\title{
Fixed point theorems for nonlinear contractive mappings in ordered $b$-metric space with auxiliary function
}

\author{
N. Seshagiri Rao ${ }^{1 *}$, K. Kalyani $^{2}$ and Belay Mitiku ${ }^{1}$
}

\begin{abstract}
Objectives: In this paper we present some fixed point theorems for self mappings satisfying generalized $(\phi, \psi)$-weak contraction condition in partially ordered complete $b$-metric spaces. The results presented over here generalize and extend some existing results in the literature. Finally, we illustrate two examples to support our results.

Result: We obtained a unique fixed point of a self mapping satisfying certain contraction condition which is involving an auxiliary function. Also, the results are presented for the existence of a common fixed point and a coincidence point for generalized $(\phi, \psi)$-weak contraction mappings in partially ordered complete $b$-metric space.
\end{abstract}

Keywords: Ordered $b$-metric space, Rational type generalized contraction mappings, Fixed points, Complete metric space

Mathematics Subject Classification: 46T99, 41A50, 54H25

\section{Introduction}

Fixed points of mappings satisfying contractive conditions in generalized metric spaces are highly useful in large number of mathematical problems of pure and applied mathematics. First, Ran and Reuings [1] have extended the result in this direction, discussed the existence of fixed points for certain maps in ordered metric space and also presented some applications to matrix linear equations. Afterwords, the result of [1] has been extended by Nieto et al. [2, 3] involving nondecreasing mappings and used their results in obtaining an unique solution of a first order differential equation. At the same time, the results regarded to generalized contractions in ordered spaces were studied by Agarwal et al. [4] and, O'Regan et al. [5]. The concept of coupled fixed points for certain mappings was first introduced by Bhaskar

*Correspondence: seshu.namana@gmail.com

1 Department of Applied Mathematics, School of Applied Natural

Sciences, Adama Science and Technology University, Post Box No.1888,

Adama, Ethiopia

Full list of author information is available at the end of the article and Lakshmikantham [6] and then applied their results to a periodic boundary value problem in acquiring an unique solution. Thereafter, the concept of coupled coincidence and common fixed point results was first initiated by Lakshmikantham and Cirić [7], which were the extensions of Bhaskar and Lakshmikantham [6] involving monotone property for a function in ordered metric spaces. More work relevant to coupled fixed point results under different contractive conditions in various spaces can be found from [8-15]. Later Singh et al. [16] obtained a coincidence and common fixed point theorems for Suzuki type hybrid contractions in ordered metric spaces and presented the corresponding applications to the results in their work. Mean while a new type of coincidence and common fixed point theorems with applications was investigated by Singh et al. [17].

$b$-metric space is one of many generalizations to an usual metric, which was first introduced by Bakhtin [18] in his work and then extensively used by Czerwik in $[19,20]$. Thereafter, lot of improvements have been 
done in finding fixed points for single, multi-valued operator in that space, the readers may refer to [21-29].

In this paper, we introduce the class of generalized $(\phi, \psi)$-weak contraction to establish an existence of a fixed point and its uniqueness of a self mapping and common fixed point, coincidence point for two self mappings in ordered complete $b$ metric space. Our results generalize Theorem 2.1 and 2.2 of [38] and the Corollaries of [39] and several comparable results in the literature [32, 35-37].

\section{Mathematical preliminaries}

We start this section with the following definitions and results which are frequently used in the main results.

Definition 1 [20] A mapping $d: P \times P \rightarrow[0,+\infty)$, where $P$ is a non-empty set is said to be a $b$-metric, if it satisfies the properties given below for any $v, \xi, \mu, \in P$ and for some $s \geq 1$,
(a) $d(v, \xi)=0$ if and if $v=\xi$,
(b) $d(v, \xi)=d(\xi, v)$,
(c) $d(v, \xi) \leq s(d(v, \mu)+d(\mu, \xi))$.

And then $(P, d, s)$ is known as a $b$-metric space.

Definition 2 [20] Let $(P, d, s)$ be a $b$-metric space. Then

(1) a sequence $\left\{v_{n}\right\}$ is said to converge to $v$ if $\lim _{n \rightarrow+\infty} d\left(v_{n}, v\right)=0$ and written as $\lim _{n \rightarrow+\infty} v_{n}=v$.

(2) $\left\{v_{n}\right\}$ is said to be a Cauchy sequence in $P$, if $\lim _{n \rightarrow+\infty} d\left(v_{n}, v_{m}\right)=0$.

(3) $(P, d, s)$ is said to be complete if every Cauchy sequence in $P$ is convergent.

Definition 3 A metric $d$ on $P$ together with a partially ordered relation $\leq$ is called a partially ordered metric space. It is denoted by $(P, d, \leq)$.

Definition 4 If the metric $d$ is complete then $(P, d, \leq)$ is called a complete partially ordered metric space.

Definition 5 Let $(P, \leq)$ be a partially ordered set. A map $S: P \rightarrow P$ is said to be monotone nondecreasing, if $S(v) \leq S(\xi)$ for all $v, \xi \in P$ with $v \leq \xi$.

Definition 6 A point $v \in A$, where $A \neq \emptyset$, a subset of $(P, d)$ is called a common fixed (coincidence ) point for two self-mappings $f$ and $S$, if $v=f v=S v(f v=S v)$.
Definition 7 Two self maps $f$ and $S$ defined over a subset $A$ of $(P, d)$ are called commuting, if $f S v=S f v$, for all $v \in A$.

Definition 8 Two self maps $f$ and $S$ defined over $A \neq \emptyset$, a subset of $(P, d)$ are called compatible, if any sequence $\left\{v_{n}\right\}$ with $\lim _{n \rightarrow+\infty} f v_{n}=\lim _{n \rightarrow+\infty} S v_{n}=\mu$, for $\mu \in A$ then $\lim _{n \rightarrow+\infty} d\left(S f v_{n}, f S v_{n}\right)=0$.

Definition 9 A pair of self mappings $(f, S)$ on $A \neq \emptyset$, a subset of $P$ is called weakly compatible, if $S f v=f S v$, when $S v=f v$ for some $v \in A$.

Definition 10 Let $f$ and $S$ be two self-mappings over $(P, \leq)$. Then $S$ is called monotone $f$ nondecreasing, if

$$
f \nu \leq f \xi \Rightarrow S v \leq S \xi \text {, for any } v, \xi \in P .
$$

Definition 11 If very two elements of $A \neq \emptyset$, a sub set of $(P, \leq)$ are comparable then $A$ is called well ordered set.

The concept of acquiring fixed points in metric space using control functions was initiated by Khan et al. [30] .

Definition 12 [30] A self map $\phi$ defined on $[0,+\infty)$ is said to be an altering distance function, if $\phi$ is continuous and monotone increasing with $\phi(t)=0$ iff $t=0$.

\section{Note 1}

(1) Let us denote the set of all altering distance functions on $[0,+\infty)$ by $\Phi$.

(2) Similarly, $\Psi$ denoted by the set of lower semicontinuous functions on $[0,+\infty)$ with $\psi(t)=0$ iff $t=0$.

Lemma 1 [31] Let $P$ be a non-empty set and $f: P \rightarrow P$ be a mapping. Then there exists a subset $E$ of $P$ such that $f E=f P$ and $f: E \rightarrow P$ is one-to-one.

In 1975, Dass and Gupta [32] proved the following fixed point result in a complete metric space.

Theorem 1 [32] Suppose $(P, d)$ is a complete metric space. Let $S: P \rightarrow P$ be a mapping such that there exist $\alpha, \beta \in[0,1)$ with $\alpha+\beta<1$ satisfying

$$
d(S v, S \xi) \leq \alpha \frac{d(\xi, S \xi)[1+d(v, S v)]}{1+d(v, \xi)}+\beta d(v, \xi)
$$

for any distinct $v, \xi \in P$. Then $S$ has a unique fixed point in $P$. 
The generalization of above result in partially ordered metric space was obtained by Cabrera et al. [33] in 2013. Later Chandok et al. [34] generalized the result of [33] by use of control functions in the space.

Again, Theorem 1 was generalized by Jaggi [35] in 1977 and proved the following

Theorem 2 [35] Suppose $(P, d)$ is a complete metric space. A self map $S$ on $P$ such that

$$
d(S v, S \xi) \leq \alpha \frac{d(\xi, S \xi) d(\nu, S v)}{d(\nu, \xi)}+\beta d(\nu, \xi)
$$

for all $\nu, \xi \in P$ with $\nu \neq \xi$, where $\alpha, \beta \in[0,1)$ with $\alpha+\beta<1$. Then $S$ has a unique fixed point in $P$.

This result was again proved by Harjani et al. [36] in a complete metric space endowed with partial order relation. Later the result of [36] was generalized by Luong et al. [37] involving altering distance functions which satisfies a weak contractive condition of, rational type auxiliary functions in ordered metric space. Thereafter, the result [37] was generalized and extended by Chandok et al. [38] in 2013 and obtained coupled, common fixed point results for weak contractive mapping in partially ordered metric space. These results were again generalized by Hieu et al. [39] in partially order $b$-metric space by involving altering distance functions.

\section{Main text}

To begin this section with the following theorem.

Theorem 3 Let $(P, d, s, \leq)$ be a complete partially ordered b-metric space with parameter $s \geq 1$. Let $S: P \rightarrow P$ be a continuous, nondecreasing mapping with regards to $\leq$ such that there exists $v_{0} \in P$ with $v_{0} \leq S v_{0}$. Suppose that

$$
\phi(s d(S v, S \xi)) \leq \phi(M(v, \xi))-\psi(M(v, \xi))
$$

where $\phi \in \Phi, \psi \in \Psi$, for any $\nu, \xi \in P$ with $\nu \leq \xi$ and

$$
\begin{aligned}
M(v, \xi)= & \max \left\{\frac{d(\xi, S \xi)[1+d(v, S v)]}{1+d(v, \xi)},\right. \\
& \left.\frac{d(\xi, S v)[1+d(v, S \xi)]}{1+d(v, \xi)}, d(v, \xi)\right\} .
\end{aligned}
$$

Then $S$ has a fixed point in $P$.

\section{Proof}

If for some $v_{0} \in P$ such that $S v_{0}=v_{0}$, then the proof is finished. Assume that $v_{0}<S v_{0}$, then construct a sequence $\left\{v_{n}\right\} \subset P$ by $v_{n+1}=S v_{n}$ for $n \geq 0$. But $S$ is nondecreasing then, we get the following by using mathematical induction

$$
\begin{aligned}
& v_{0}<S v_{0}=v_{1} \leq S v_{1}=v_{2} \leq \ldots \ldots \ldots \ldots . . \leq S v_{n-1} \\
& =v_{n} \leq S v_{n}=v_{n+1} \leq \ldots \ldots . .
\end{aligned}
$$

If for some $n_{0} \in \mathbb{N}$ such that $v_{n_{0}}=v_{n_{0}+1}$ then from (5), $v_{n_{0}}$ is a fixed point of $S$ and we have nothing to prove. Suppose that $v_{n} \neq v_{n+1}$, i.e., $d\left(v_{n}, v_{n+1}\right)>0$, for all $n \geq 1$. Since $v_{n}>v_{n-1}$, for any $n \geq 1$ and then by (3), we have

$$
\begin{aligned}
\phi\left(d\left(v_{n}, v_{n+1}\right)\right) & =\phi\left(d\left(S v_{n-1}, S v_{n}\right)\right) \leq \phi\left(s d\left(S v_{n-1}, S v_{n}\right)\right) \\
& \leq \phi\left(M\left(v_{n-1}, v_{n}\right)\right)-\psi\left(M\left(v_{n-1}, v_{n}\right)\right),
\end{aligned}
$$

where

$$
\begin{aligned}
M\left(v_{n-1}, v_{n}\right)= & \max \left\{\frac{d\left(v_{n}, S v_{n}\right)\left[1+d\left(v_{n-1}, S v_{n-1}\right)\right]}{1+d\left(v_{n-1}, v_{n}\right)},\right. \\
& \frac{d\left(v_{n}, S v_{n-1}\right)\left[1+d\left(v_{n-1}, S v_{n}\right)\right]}{1+d\left(v_{n-1}, v_{n}\right)} \\
& \left.d\left(v_{n-1}, v_{n}\right)\right\} \\
= & \max \left\{d\left(v_{n}, v_{n+1}\right), 0, d\left(v_{n-1}, v_{n}\right)\right\} \\
= & \max \left\{d\left(v_{n}, v_{n+1}\right), d\left(v_{n-1}, v_{n}\right)\right\}
\end{aligned}
$$

which implies that

$$
\begin{aligned}
\phi\left(d\left(v_{n}, v_{n+1}\right)\right) \leq & \phi\left(\max \left\{d\left(v_{n}, v_{n+1}\right), d\left(v_{n-1}, v_{n}\right)\right\}\right) \\
& -\psi\left(\max \left\{d\left(v_{n}, v_{n+1}\right), d\left(v_{n-1}, v_{n}\right)\right\}\right) .
\end{aligned}
$$

If $\max \left\{d\left(v_{n}, v_{n+1}\right), d\left(v_{n-1}, v_{n}\right)\right\}=d\left(v_{n}, v_{n+1}\right)$ for some $n \geq 1$, then from (8), we get

$$
\begin{aligned}
\phi\left(d\left(v_{n}, v_{n+1}\right)\right) \leq & \phi\left(d\left(v_{n}, v_{n+1}\right)\right) \\
& -\psi\left(d\left(v_{n}, v_{n+1}\right)\right)<\phi\left(d\left(v_{n}, v_{n+1}\right)\right),
\end{aligned}
$$

which is a contradiction under (9). Thus, $\max \left\{d\left(v_{n}, v_{n+1}\right)\right.$, $\left.d\left(v_{n-1}, v_{n}\right)\right\}=d\left(v_{n-1}, v_{n}\right)$ for $n \geq 1$ and hence from (8) again we have

$$
\begin{aligned}
\phi\left(d\left(v_{n}, v_{n+1}\right)\right) \leq & \phi\left(d\left(v_{n}, v_{n-1}\right)\right) \\
& -\psi\left(d\left(v_{n}, v_{n-1}\right)\right)<\phi\left(d\left(v_{n}, v_{n-1}\right)\right) .
\end{aligned}
$$

Therefore, the sequence $\left\{d\left(v_{n}, v_{n-1}\right)\right\}$ for $n \geq 1$ is a monotone non-increasing and bounded. From a result, we have

$$
\lim _{n \rightarrow+\infty} d\left(v_{n}, v_{n-1}\right)=\rho \geq 0 .
$$

Now, taking the upper limit on both sides of (6), we obtain

$$
\begin{aligned}
\phi(\rho) & \leq \phi(\rho)-\lim _{n \rightarrow+\infty} \inf \psi\left(d\left(v_{n}, v_{n-1}\right)\right) \\
& \leq \phi(\rho)-\psi(\rho)<\phi(\rho)
\end{aligned}
$$

which is a contradiction under (12). Thus, $\rho=0$. Hence, $d\left(v_{n}, v_{n-1}\right) \rightarrow 0$ as $n \rightarrow+\infty$. 
Next, we prove that $\left\{v_{n}\right\}$ is a Cauchy sequence in $P$. Assume opposite that $\left\{v_{n}\right\}$ is not a Cauchy sequence. Then for some $\epsilon>0$, we can get two subsequences $\left\{v_{m_{j}}\right\}$ and $\left\{v_{n_{j}}\right\}$ of $\left\{v_{n}\right\}$, where $n_{j}$ is the smallest index such that

$$
n_{j}>m_{j}>j, \quad d\left(v_{m_{j}}, v_{n_{j}}\right) \geq \epsilon
$$

and

$$
d\left(v_{m_{j}}, v_{n_{j}-1}\right)<\epsilon .
$$

Applying triangular inequality in (13), we get

$$
\begin{aligned}
\epsilon & \leq d\left(v_{m_{j}}, v_{n_{j}}\right) \leq s d\left(v_{m_{j}}, v_{n_{j}-1}\right)+s d\left(v_{n_{j}-1}, v_{n_{j}}\right) \\
& \leq s^{2} d\left(v_{m_{j}}, v_{m_{j}-1}\right)+s^{2} d\left(v_{m_{j}-1}, v_{n_{j}-1}\right)+s d\left(v_{n_{j}-1}, v_{n_{j}}\right)
\end{aligned}
$$

Furthermore,

$$
\begin{aligned}
& d\left(v_{m_{j}-1}, v_{n_{j}-1}\right) \leq s d\left(v_{m_{j}-1}, v_{m_{j}}\right) \\
& +s d\left(v_{m_{j}}, v_{n_{j}-1}\right) \leq s d\left(v_{m_{j}-1}, v_{m_{j}}\right)+s \epsilon
\end{aligned}
$$

Letting $j \rightarrow+\infty$ in Eqs. (15), (16) and combining together, we obtain the following inequality

$$
\frac{\epsilon}{s^{2}} \leq \lim _{j \rightarrow+\infty} \sup d\left(v_{m_{j}-1}, v_{n_{j}-1}\right) \leq s \epsilon
$$

Similarly, we can get the following inequalities by using triangular inequality

$$
\frac{\epsilon}{s^{2}} \leq \lim _{j \rightarrow+\infty} \inf d\left(v_{m_{j}-1}, v_{n_{j}-1}\right) \leq s \epsilon,
$$

and

$$
\frac{\epsilon}{s} \leq \lim _{j \rightarrow+\infty} \sup d\left(v_{m_{j}-1}, v_{n_{j}}\right) \leq s \epsilon^{2} .
$$

Let

$$
\begin{aligned}
M\left(v_{m_{j}-1}, v_{n_{j}-1}\right) & \max \left\{\frac{d\left(v_{n_{j}-1}, S v_{n_{j}-1}\right)\left[1+d\left(v_{m_{j}-1}, S v_{m_{j}-1}\right)\right]}{1+d\left(v_{m_{j}-1}, v_{n_{j}-1}\right)},\right. \\
& \left.\frac{d\left(v_{n_{j}-1}, S v_{m_{j}-1}\right)\left[1+d\left(v_{m_{j}-1}, S v_{n_{j}-1}\right)\right]}{1+d\left(v_{m_{j}-1}, v_{n_{j}-1}\right)}, d\left(v_{m_{j}-1}, v_{n_{j}-1}\right)\right\} \\
= & \max \left\{\frac{d\left(v_{n_{j}-1}, v_{n_{j}}\right)\left[1+d\left(v_{m_{j}-1}, v_{m_{j}}\right)\right]}{1+d\left(v_{m_{j}-1}, v_{n_{j}-1}\right)},\right. \\
& \left.\frac{d\left(v_{n_{j}-1}, v_{m_{j}}\right)\left[1+d\left(v_{m_{j}-1}, v_{n_{j}}\right)\right]}{1+d\left(v_{m_{j}-1}, v_{n_{j}-1}\right)}, d\left(v_{m_{j}-1}, v_{n_{j}-1}\right)\right\} .
\end{aligned}
$$

From (20), we obtain the following inequalities

$$
\frac{\epsilon}{s^{2}} \leq \lim _{j \rightarrow+\infty} \sup M\left(v_{m_{j}-1}, v_{n_{j}-1}\right) \leq s \epsilon .
$$

and

$$
\frac{\epsilon}{s^{2}} \leq \lim _{j \rightarrow+\infty} \inf M\left(v_{m_{j}-1}, v_{n_{j}-1}\right) \leq s \epsilon
$$

Form (5), we have $v_{m_{j}-1}<v_{n_{j}-1}$, then

$$
\begin{aligned}
& \phi\left(s d\left(v_{m_{j}}, v_{n_{j}}\right)\right)=\phi\left(s d\left(S v_{m_{j}-1}, S v_{n_{j}-1}\right)\right) \\
& \leq \phi\left(M\left(v_{m_{j}-1}, v_{n_{j}-1}\right)\right)-\psi\left(M\left(v_{m_{j}-1}, v_{n_{j}-1}\right)\right) .
\end{aligned}
$$

Now, letting $j \rightarrow+\infty$ and use of (21), (22), we obtain

$$
\begin{aligned}
\phi(s \epsilon) \leq & \phi\left(s \lim _{j \rightarrow+\infty} d\left(v_{m_{j}}, v_{n_{j}}\right)\right) \\
\leq & \phi\left(\lim _{j \rightarrow+\infty} \sup M\left(v_{m_{j}-1}, v_{n_{j}-1}\right)\right) \\
& -\lim _{j \rightarrow+\infty} \inf \psi\left(M\left(v_{m_{j}-1}, v_{n_{j}-1}\right)\right) \\
\leq & \phi(s \epsilon)-\psi\left(\lim _{j \rightarrow+\infty} \inf M\left(v_{m_{j}-1}, v_{n_{j}-1}\right)\right) \\
< & \phi(s \epsilon)
\end{aligned}
$$

this is a contradiction under (24). Hence, $\left\{v_{n}\right\}$ is a Cauchy sequence and converges to some $\mu \in P$ as $P$ is complete. Also, the continuity of $S$ implies that

$$
S \mu=S\left(\lim _{n \rightarrow+\infty} v_{n}\right)=\lim _{n \rightarrow+\infty} S v_{n}=\lim _{n \rightarrow+\infty} v_{n+1}=\mu .
$$

Therefore, $\mu$ is a fixed point of $S$ in $P$

By weakening the continuity property of a map $S$ in Theorem 3, we have the following result.

Theorem 4 In Theorem 3, if $P$ has a property that, the sequence $\left\{v_{n}\right\}$ is a nondecreasing such that $v_{n} \rightarrow v$, implies that $v_{n} \leq v$, for all $n \in \mathbb{N}$, i.e., $v=\sup v_{n}$ then a non continuous map $S$ has a fixed point in $P$.

\section{Proof}

From Theorem 3, we take the same sequence $\left\{v_{n}\right\}$ in $P$ such that $v_{0} \leq v_{1} \leq v_{2} \leq v_{3} \leq \ldots \ldots . . \leq v_{n} \leq v_{n+1} \leq \ldots . . . .$. . i.e., the sequence $\left\{v_{n}\right\}$ is nondecreasing and converges to some $v$ in $P$. Thus, from the hypotheses we have $v_{n} \leq v$, for any $n \in \mathbb{N}$, implies that $v=\sup v_{n}$.

Next, we prove that $v$ is a fixed point of $S$ in $P$, that is $S v=v$. Suppose $S v \neq v$, that is $d(S v, v) \neq 0$. Let 


$$
\begin{aligned}
M\left(v_{n}, v\right)= & \max \left\{\frac{d(v, S v)\left[1+d\left(v_{n}, S v_{n}\right)\right]}{1+d\left(v_{n}, v\right)},\right. \\
& \left.\frac{d\left(v, S v_{n}\right)\left[1+d\left(v_{n}, S v\right)\right]}{1+d\left(v_{n}, v\right)}, d\left(v_{n}, v\right)\right\} \\
= & \max \left\{\frac{d(v, S v)\left[1+d\left(v_{n}, v_{n+1}\right)\right]}{1+d\left(v_{n}, v\right)},\right. \\
& \left.\frac{d\left(v, v_{n+1}\right)\left[1+d\left(v_{n}, S v\right)\right]}{1+d\left(v_{n}, v\right)}, d\left(v_{n}, v\right)\right\}
\end{aligned}
$$

Letting $n \rightarrow+\infty$ and from $\lim _{n \rightarrow+\infty} v_{n}=v$, we get

$$
\lim _{n \rightarrow+\infty} M\left(v_{n}, v\right)=\max \{d(v, S v), 0,0\}=d(v, S v) .
$$

We know that $v_{n} \leq v$, for all $n$ then from contraction condition (3), we get

$$
\begin{aligned}
& \phi\left(d\left(v_{n+1}, S v\right)\right)=\phi\left(d\left(S v_{n}, S v\right)\right. \\
& \leq \phi\left(s d\left(S v_{n}, S v\right) \leq \phi\left(M\left(v_{n}, v\right)\right)-\psi\left(M\left(v_{n}, v\right)\right)\right.
\end{aligned}
$$

Letting $n \rightarrow+\infty$ and use of (27), we get

$$
\phi(d(v, S v)) \leq \phi(d(v, S v))-\psi(d(v, S v))<\phi(d(v, S v))
$$

which is wrong under (29). Thus, $S v=v$, that is $S$ has a fixed point $v$ in $P$.

The uniqueness of, an existing fixed point in Theorems 3 and 4 can get, if $P$ has the following property:

For any $v, \xi \in P$, there exists $v \in P$ such that $v \leq v$ and $v \leq \xi$.

Theorem 5 If P satisfies the above mentioned condition in Theorem 3 (or Theorem 4) then $S$ has a unique fixed point.

Proof

From Theorem 3 (or Theorem 4), we conclude that S has a nonempty set of fixed points. Suppose that $v^{*}$ and $\xi^{*}$ be two fixed points of S then, we claim that $v^{*}=\xi^{*}$. Suppose that $v^{*} \neq \xi^{*}$, then from the hypotheses we have

$$
\begin{aligned}
\phi\left(d\left(S v^{*}, S \xi^{*}\right)\right) & \leq \phi\left(s d\left(S v^{*}, S \xi^{*}\right)\right) \\
& \leq \phi\left(M\left(v^{*}, \xi^{*}\right)\right)-\psi\left(M\left(v^{*}, \xi^{*}\right)\right)
\end{aligned}
$$

where

$$
\begin{aligned}
M\left(v^{*}, \xi^{*}\right)= & \max \left\{\frac{d\left(\xi^{*}, S \xi^{*}\right)\left[1+d\left(v^{*}, S v^{*}\right)\right]}{1+d\left(v^{*}, \xi^{*}\right)},\right. \\
& \left.\frac{d\left(\xi^{*}, S v^{*}\right)\left[1+d\left(v^{*}, S \xi^{*}\right)\right]}{1+d\left(v^{*}, \xi^{*}\right)}, d\left(v^{*}, \xi^{*}\right)\right\} \\
= & \max \left\{\frac{d\left(\xi^{*}, \xi^{*}\right)\left[1+d\left(v^{*}, v^{*}\right)\right]}{1+d\left(v^{*}, \xi^{*}\right)},\right. \\
& \left.\frac{d\left(\xi^{*}, v^{*}\right)\left[1+d\left(v^{*}, \xi^{*}\right)\right]}{1+d\left(v^{*}, \xi^{*}\right)}, d\left(v^{*}, \xi^{*}\right)\right\} \\
= & \max \left\{0, d\left(\xi^{*}, v^{*}\right), d\left(v^{*}, \xi^{*}\right)\right\} \\
= & d\left(v^{*}, \xi^{*}\right) .
\end{aligned}
$$

Then by Eq. (30), we have

$$
\begin{aligned}
\phi\left(d\left(v^{*}, \xi^{*}\right)\right) & =\phi\left(d\left(S v^{*}, S \xi^{*}\right)\right) \\
& \leq \phi\left(d\left(v^{*}, \xi^{*}\right)\right)-\psi\left(d\left(v^{*}, \xi^{*}\right)\right) \\
& <\phi\left(d\left(v^{*}, \xi^{*}\right)\right)
\end{aligned}
$$

which is a contradiction under (32). Hence, $v^{*}=\xi^{*}$.

The proof is completed.

Now, we have the results below, which are the generalizations of Theorems-2.1 and 2.2 of [38] and the Corollaries-2.1 and 2.2 of [39] in the space.

Corollary 1 Let $(P, d, s, \leq)$ be a partially ordered $b$-metric space with a parameter s. Suppose $S, f: P \rightarrow P$ are continuous mappings such that

$\left(C_{1}\right)$. for some $\psi \in \Psi$ and $\phi \in \Phi$ with

$$
\phi(s d(S v, S \xi)) \leq \phi\left(M_{f}(v, \xi)\right)-\psi\left(M_{f}(v, \xi)\right)
$$

for any $v, \xi \in P$ such that $f \nu \leq f \xi$ and

$$
\begin{aligned}
M_{f}(\nu, \xi)= & \max \left\{\frac{d(f \xi, S \xi)[1+d(f v, S v)]}{1+d(f v, f \xi)},\right. \\
& \left.\frac{d(f \xi, S v)[1+d(f v, S \xi)]}{1+d(f v, f \xi)}, d(f \nu, f \xi)\right\},
\end{aligned}
$$

$\left(C_{2}\right) \quad S P \subset f P$ and $f P$ is a complete subspace of $P$,

$\left(C_{3}\right) \quad S$ is a monotone $f$-non decreasing mapping,

$\left(C_{4}\right) \quad S$ and $f$ are compatible.

If for some $v_{0} \in P$ such that $f v_{0} \leq S v_{0}$, then $S$ and $f$ have a coincidence point in $P$. 
Proof

By using lemma 1, we obtain a complete subspace $f E$ of $P$, where $E \subset P$ and $f$ is one-to-one self mapping on $P$. By Corollary 2.1 of [39], we have a sequence $\left\{f v_{n}\right\} \subset f E$ for some $v_{0} \in E$ with $f v_{n+1}=S v_{n}=g\left(f v_{n}\right)$, for $n \geq 0$, where $g$ is a self-mapping on $f E$ with $g(f v)=S v, v \in E$. So, from the hypotheses we have

$$
\phi(s d(g(f v), g(f \xi))) \leq \phi\left(M_{f}(\nu, \xi)\right)-\psi\left(M_{f}(\nu, \xi)\right)
$$

for all $v, \xi \in P$ with $f v \leq f \xi$ and,

$$
\begin{aligned}
M_{f}(v, \xi)= & \max \left\{\frac{d(f \xi, g(f \xi))[1+d(f v, g(f v))]}{1+d(f v, f \xi)},\right. \\
& \left.\frac{d(f \xi, g(f v))[1+d(f v, g(f \xi))]}{1+d(f v, f \xi)}, d(f v, f \xi)\right\} .
\end{aligned}
$$

From the same argument in Theorem 3, $\left\{f v_{n}\right\}$ is a Cauchy sequence and which converges for some $v \in f E$. Thus, by the compatibility of $S$ and $f$, we obtain

$$
\lim _{n \rightarrow+\infty} d\left(f\left(S v_{n}\right), S\left(f v_{n}\right)\right)=0 .
$$

Further, by use of triangular inequality,

$$
\begin{aligned}
d(S v, f v) \leq & s d\left(S v, S\left(f v_{n}\right)\right)+s^{2} d\left(S\left(f v_{n}\right), f\left(S v_{n}\right)\right) \\
& +s^{2} d\left(f\left(S v_{n}\right), f v\right) .
\end{aligned}
$$

Finally, we arrive at $d(S v, f v)=0$ as $n \rightarrow+\infty$ in (38). Therefore, $v$ is a coincidence point for $S$ and $f$ in $P$.

Replace the condition, weakly compatible instead of (C4) in Corollary 1, we obtain the following result.

Corollary 2 If $P$ has the property in Corollary 1 instead of the compatibility for $S$ and $f$ that, for any nondecreasing sequence $\left\{f v_{n}\right\} \subset P$ such that $\lim _{n \rightarrow+\infty} f v_{n}=f v$ implies that $f v_{n} \leq f v$ for all $n \in \mathbb{N}$, that is $f v=\sup f v_{n}$. Then $S$ and $f$ have a common fixed point in $P$, if for some coincidence point $\mu$ of $S$ and $f$ with $f \mu \leq f(f \mu)$. Furthermore, the set of common fixed points of $S$ and $f$ is well ordered if and only if $S$ and f have one and only one common fixed point.

\section{Proof}

It is obvious from Corollary 1 and Theorem 4 that $S$ and $f$ have a coincidence point in $P$, as $f \mu=g(f \mu)=S \mu$ for some $\mu$ in $P$.

Next, assume that a pair of mappings $(S, f)$ is weakly compatible and let $\vartheta$ be an element in $P$ such that $\vartheta=S \mu=f \mu$. Then, $S \vartheta=S(f \mu)=f(S \mu)=f \vartheta$. Let

$$
\begin{aligned}
M(\mu, \vartheta)= & \max \left\{\frac{d(f \vartheta, S \vartheta)[1+d(f \mu, S \mu)]}{1+d(f \mu, f \vartheta)},\right. \\
& \left.\frac{d(f \vartheta, S \mu)[1+d(f \mu, S \vartheta)]}{1+d(f \mu, f \vartheta)}, d(f \mu, f \vartheta)\right\} \\
= & \max \{0, d(S \mu, S \vartheta)\} \\
= & d(S \mu, S \vartheta) .
\end{aligned}
$$

then from contraction condition, we have

$$
\begin{aligned}
\phi(d(S \mu, S \vartheta)) & \leq \phi(M(\mu, \vartheta))-\psi(M(\mu, \vartheta)) \\
& \leq \phi(d(S \mu, S \vartheta))-\psi(d(S \mu, S \vartheta)) .
\end{aligned}
$$

Hence, we get $d(S \mu, S \vartheta)=0$ by the property of $\psi$. Therefore, $S \vartheta=f \vartheta=\vartheta$.

Eventually, by following Theorem 5, we deduce that $S$ and $f$ have one and only one common fixed point if and only if the set of common fixed points of $S$ and $f$ is well ordered.

We illustrate the usefulness of the obtained results in different cases such as continuity and discontinuity of a metric $d$ in a space $P$.

\section{Example 1}

Define a metric $d: P \rightarrow P$ as below and $\leq$ be an usual order in $P$, where $P=\{1,2,3,4,5\}$

$$
\begin{aligned}
& d(v, \xi)=d(\xi, v)=0, \text { if } v, \xi=1,2,3,4,5 \text { and } v=\xi . \\
& d(v, \xi)=d(\xi, v)=1, \text { if } v, \xi=1,2,3,4 \text { and } v \neq \xi . \\
& d(v, \xi)=d(\xi, v)=6, \text { if } v=1,2,3 \text { and } \xi=5 . \\
& d(v, \xi)=d(\xi, v)=12, \text { if } v=4 \text { and } \xi=5 .
\end{aligned}
$$

Define a map $S: P \rightarrow P$ by $S 1=S 2=S 3=S 4=1, S 5=3$ and let $\phi(t)=\frac{t}{2}, \psi(t)=\frac{t}{3}$ for $t \in[0,+\infty)$. Then $S$ has a fixed point in $P$.

Proof

It is apparent that, $(P, d, s, \leq)$ is a complete partially ordered b-metric space for $s=2$. Consider the possible cases for $v, \xi$ in $P$ :

Case 1 Suppose $v, \xi \in\{1,2,3,4\}$ and $v<\xi$, thence,

$$
\phi(2 d(S v, S \xi))=0 \leq \phi(M(\nu, \xi))-\psi(M(v, \xi)) .
$$

Case 2 Suppose that $v \in\{1,2,3,4\}$ and $\xi=5$, then $d(S v, S \xi)=d(1,3)=1, M(4,5)=12$ and $M(v, 5)=6$, for $v \in\{1,2,3\}$. Therefore, we have the following inequality, 


$$
\phi(2 d(S v, S \xi)) \leq \frac{M(v, \xi)}{6}=\phi(M(v, \xi))-\psi(M(v, \xi)) .
$$

Thus, condition (3) of Theorem 3 holds. Furthermore, the remaining assumptions in Theorem 3 are fulfilled. Hence, $S$ has a fixed point in $P$ as Theorem 3 is appropriate to $S, \phi, \psi$ and $(P, d, s, \leq)$.

\section{Example 2}

A metric $d: P \rightarrow P$, where $P=\left\{0,1, \frac{1}{2}, \frac{1}{3}, \frac{1}{4}, \ldots \ldots . . \frac{1}{n}, \ldots ..\right\}$ with usual order $\leq$ is defined as follows

$$
d(v, \xi)= \begin{cases}0, & \text { if } v=\xi \\ 1 & , \text { if } v \neq \xi \in\{0,1\} \\ |v-\xi| & , \text { if } v, \xi \in\left\{0, \frac{1}{2 n}, \frac{1}{2 m}: n \neq m \geq 1\right\} \\ 5 & , \text { otherwise. }\end{cases}
$$

A map $S: P \rightarrow P$ be such that $S 0=0, S \frac{1}{n}=\frac{1}{9 n}$ for all $n \geq 1$ and let $\phi(t)=t, \psi(t)=\frac{3 t}{4}$ for $t \in[0,+\infty)$. Then $S$ has a fixed point in $P$.

\section{Proof}

It's obvious that for $s=\frac{9}{4},(P, d, s, \leq)$ is a complete partially ordered b-metric space and also by definition, $d$ is discontinuous b-metric space. Now, for $v, \xi \in P$ with $\nu<\xi$ then consider the following cases:

Case 1 If $v=0$ and $\xi=\frac{1}{n}, n \geq 1$, then $d(S v, S \xi)=$ $d\left(0, \frac{1}{9 n}\right)=\frac{1}{9 n}$ and $M(v, \xi)=\frac{1}{n}$ or $M(v, \xi)=\{1,5\}$. Therefore, we have

$$
\phi\left(\frac{9}{4} d(S v, S \xi)\right) \leq \frac{M(\nu, \xi)}{4}=\phi(M(v, \xi))-\psi(M(v, \xi)) .
$$

Case 2 If $v=\frac{1}{m}$ and $\xi=\frac{1}{n}$ with $m>n \geq 1$, then

$$
\begin{aligned}
& d(S v, S \xi)=d\left(\frac{1}{9 m}, \frac{1}{9 n}\right) \text { and } \\
& M(v, \xi) \geq \frac{1}{n}-\frac{1}{m} \text { or } M(v, \xi)=5 .
\end{aligned}
$$

Therefore,

$$
\phi\left(\frac{9}{4} d(S v, S \xi)\right) \leq \frac{M(v, \xi)}{4}=\phi(M(v, \xi))-\psi(M(v, \xi)) .
$$

Hence, condition (3) of Theorem 3 and remaining assumptions are satisfied. Thus, $S$ has a fixed point in $P$.

\section{Limitations}

In this manuscript, we obtained a fixed point for generalized $(\phi, \psi)$-weak contraction mapping in a complete partially ordered $b$-metric space. We establish that this mapping has a unique fixed point under ordered relation in a space. Also, we have investigated a common fixed point and a coincidence point for mappings satisfying generalized $(\phi, \psi)$-weak contraction condition in the same space. These results generalize and extended some well known results in the literature. At the end some illustrations are given to support our results.

\section{Acknowledgements}

The authors do thankful to the editor and anonymous reviewers for their valuable suggestion which improve the content of the article.

\section{Authors' contributions}

All authors contributed equally to write of this paper. All authors read and approved the final manuscript.

\section{Funding}

Not applicable.

\section{Availability of data and materials} Not applicable.

Ethics approval and consent to participate Not applicable.

\section{Consent for publication}

Not applicable.

\section{Competing interests}

The authors declare that they have no competing interests.

\section{Author details}

${ }^{1}$ Department of Applied Mathematics, School of Applied Natural Sciences, Adama Science and Technology University, Post Box No.1888, Adama, Ethiopia. ${ }^{2}$ Department of Mathematics, Vignan's Foundation for Science, Technology \& Research, Vadlamudi, Andhra Pradesh 522213, India.

Received: 16 May 2020 Accepted: 9 September 2020 Published online: 22 September 2020

\section{References}

1. Ran ACM, Reurings MCB. A fixed point theorem in partially ordered sets and some applications to matrix equations. Proc Am Math Soc. 2004:132:1435-43. https://doi.org/10.1090/S0002-9939-03-07220-4.

2. Nieto JJ, Loṕez RR. Contractive mapping theorems in partially ordered sets and applications to ordinary differential equations. Order. 2005;22:223-39. https://doi.org/10.1007/s1 1083-005-9018-5.

3. Nieto JJ, Loṕez RR. Existence and uniqueness of fixed point in partially ordered sets and applications to ordinary differential equations. Acta Math Sinica Engl. Ser. 2007;23(12):2205-12. https://doi.org/10.1007/s1011 4-005-0769-0.

4. Agarwal RP, El-Gebeily MA, O'Regan D. Generalized contractions in partially ordered metric spaces. Appl Anal. 2008;87:1-8. https://doi. org/10.1080/00036810701714164.

5. O'Regan D, Petrutel A. Fixed point theorems for generalized contractions in ordered metric spaces. J Math Anal Appl. 2008;341:1241-52.

6. Bhaskar TG, Lakshmikantham V. Fixed point theorems in partially ordered metric spaces and applications. Nonlinear Anal. 2006;65:1379-93. https:// doi.org/10.1016/j.na.2005.10.017. 
7. Lakshmikantham V Ćirić LJ. Coupled fixed point theorems for nonlinear contractions in partially ordered metric spaces. Nonlinear Anal. 2009;70:4341-9. https://doi.org/10.1016/j.na.2008.09.020.

8. Cho YJ, Shah MH, Hussain N. Coupled fixed points of weakly F-contractive mappings in topological spaces. Appl Math Lett. 2011;24:1185-90. https ://doi.org/10.1016/j.aml.2011.02.004

9. Ćirić LJ, Cakic N, Rajović M, Ume JS. Monotone generalized nonlinear contractions in partially ordered metric spaces. Fixed Point Theory Appl. 2008:2008:11.

10. Graily E, Vaezpour SM, Saadati R, Cho YJ. Generalization of fixed point theorems in ordered metric spaces concerning generalized distance. Fixed Point Theory Appl. 2011;2011:30. https://doi. org/10.1186/1687-1812-2011-30.

11. Karapinar E. Couple fixed point theorems for nonlinear contractions in cone metric spaces. Comput Math Appl. 2010;59(12):3656-68. https:// doi.org/10.1016/j.camwa.2010.03.062.

12. Sabetghadam F, Masiha HP, Sanatpour AH. Some coupled fixed point theorems in cone metric spaces. Fixed point Theory Appl. 2009;2009:1-8.

13. Samet B, Yazidi H. Coupled fixed point theorems in partially ordered $\epsilon$ -chainable metric spaces. J Math Comput Sci. 2010;1:142-51.

14. Sintunavarat W, Cho YJ, Kumam P. Common fixed point theorems for c-distance in ordered cone metric spaces. Comput Math Appl. 2011;62:1969-78. https://doi.org/10.1016/j.camwa.2011.06.040.

15. Shatanawi W. Partially ordered cone metric spaces and coupled fixed point results. Comput Math Appl. 2010;60:2508-15. https://doi. org/10.1016/j.camwa.2010.08.074.

16. Singh SL, Kamal R, Sen MDL. Coincidence and common fixed point theorems for Suzuki type hybrid contractions and applications. Fixed Point Theory Appl. 2014;2014(1):147. https://doi. org/10.1186/1687-1812-2014-147.

17. Singh SL, Kamal R, Sen MDL. Renu Chugh, A new type of coincidence and common fixed point theorems with applications. Abstr Appl Anal. 2014;2014:642378. https://doi.org/10.1155/2014/642378 (11 Pages).

18. Bakhtin IA. The contraction principle in quasimetric spaces. Func An Ulianowsk Gos Fed Ins. 1989;30:26-37.

19. Czerwik S. Contraction mappings in b-metric spaces. Acta Math Univ Ostrav. 1993;1:5-11

20. Czerwik S. Nonlinear set-valued contraction mappings in b-metric spaces. Atti Semin Mat Fis Univ Modena. 1998;46(2):263-76.

21. Aghajani A, Arab R. Fixed points of $(\psi, \phi, \theta)$-contractive mappings in partially ordered $b$-metric spaces and application to quadratic integral equations. Fixed Point Theory Appl. 2013:2013(245):1-20.

22. Aghajani A, Abbas M, Roshan JR. Common fixed point of generalized weak contractive mappings in partially ordered $b$-metric spaces. Math Slovaca. 2014;64(4):941-60.

23. Huang $\mathrm{H}, \mathrm{X}$ U S. Fixed point theorems of contractive mappings in cone $b$ -metric spaces and applications. Fixed Point Theory Appl. 2012;2012:1-8.

24. Hussain N, Parvaneh V, Roshan JR, Kadelburg Z. Fixed points of cyclic weakly $(\psi, \phi, L, A, B)$-contractive mappings in ordered $b$-metric spaces with applications. Fixed Point Theory Appl. 2013;2013(256):1-18.
25. Parvaneh V, Roshan JR, Radenović S. Existence of tripled coincidence points in ordered $b$-metric spaces and an application to a system of integral equations. Fixed Point Theory Appl. 2013;2013(130):1-19.

26. Roshan JR, Parvaneh V, Altun I. Some coincidence point results in ordered b-metric spaces and applications in a system of integral equations. Appl Math Comput. 2014;226:725-37.

27. Roshan JR, Parvaneh V, Kadelburg Z. Common fixed point theorems for weakly isotone increasing mappings in ordered $b$-metric spaces. J Nonlinear Sci Appl. 2014;7:229-45.

28. Roshan JR, Parvaneh V, Sedghi S, Shobkolaei N, Shatanawi W. Common fixed points of almost generalized $(\psi, \phi)_{s}$-contractive mappings in ordered $b$-metric spaces. Fixed Point Theory Appl. 2013;2013(159):1-23.

29. Shatanawi W, Pitea A, Lazović R. Contraction conditions using comparison functions on $b$-metric spaces. Fixed Point Theory Appl. 2014;2014(135):1-11

30. Khan MS, Swaleh M, Sessa S. Fixed points theorems by altering distances between the points. Bull Aust Math Soc. 1984;30:1-9.

31. Haghi RH, Rezapour S, Shahzad N. Some fixed point generalizations are not real generalizations. Nonlinear Anal. 2011;74:1799-803.

32. Dass BK, Gupta S. An extension of Banach contraction principle through rational expressions. Inidan J Pure Appl Math. 1975;6:1455-8.

33. Cabrera I, Harjani J, Sadarangani K. A fixed point theorem for contractions of rational type in partially ordered metric spaces. Ann Univ Ferrara. 2013:59:251-8.

34. Chandok S, Choudhury BS, Metiya N. Fixed point results in ordered metric spaces for rational type expressions with auxiliary functions. J Egypt Math Soc. 2015;23:95-101.

35. Jaggi DS. Some unique fixed point theorems. Indian J Pure Appl Math. 1977:8:223-30

36. Harjani J, Loṕez B, Sadarangani K. A fixed point theorem for mappings satisfying a contractive condition of rational type on a partially ordered metric space. Abstr. Appl. Anal. 2010;2010:1-8.

37. Luong NV, Thuan NX. Fixed point theorem for generalized weak contractions satisfying rational expressions in ordered metric spaces. Fixed Point Theory Appl. 2011;2011(46):1-10.

38. Chandok S, Karapinar E. Common fixed point of generalized rational type contraction mappings in partially ordered metric spaces. Thai J. Math. 2013:11(2):251-60.

39. Hieu NT, Dung NV. Some fixed point results for generalized rational type contraction mappings in partially ordered $b$-metric space. Facta Univ Ser Math Inform. 2015;30(1):49-66.

\section{Publisher's Note}

Springer Nature remains neutral with regard to jurisdictional claims in published maps and institutional affiliations. 